\title{
Activity of a novel sulfonamide compound 2-nitro-N-(pyridin-2-ylmethyl)benzenesulfonamide against Leishmania donovani
}

This article was published in the following Dove Press journal:

Drug Design, Development and Therapy

26 May 2016

Number of times this article has been viewed

\author{
Manas R Dikhit, ',* \\ Bidyut Purkait, I,* Ruby \\ Singh,' Bikash Ranjan \\ Sahoo, ${ }^{2}$ Ashish Kumar, ${ }^{\prime}$ \\ Rajiv K Kar, ${ }^{3}$ Md Yousuf \\ Ansari, ${ }^{1,4}$ Savita Saini,, \\ Kumar Abhishek,' Ganesh \\ C Sahoo,' Sushmita Das, ${ }^{6}$ \\ Pradeep Das' \\ 'Department of Molecular \\ Parasitology and Biomedical \\ Informatics, Rajendra Memorial \\ Research Institute of Medical \\ Sciences, Indian Council of Medical \\ Research, Agamkuan, Patna, Bihar, \\ India; 'Laboratory of Molecular \\ Biophysics, Institute for Protein \\ Research, Osaka University, Japan; \\ ${ }^{3}$ Biomolecular Nuclear Magnetic \\ Resonance and Drug Design \\ Laboratory, Department of Biophysics, \\ Bose Institute, Kolkata, West Bengal, \\ ${ }^{4}$ Department of Pharmacoinformatics, \\ ${ }^{5}$ Department of Biotechnology, \\ National Institute of Pharmaceutical \\ Education and Research, Hajipur, \\ ${ }^{6}$ Department of Microbiology, All India \\ Institute of Medical Sciences, Patna, \\ Bihar, India \\ *These authors contributed equally \\ to this work
}

Correspondence: Pradeep Das Department of Molecular Parasitology and Bioinformatics, Rajendra Memorial Research Institute of Medical Sciences, Indian Council of Medical Research,

Agamkuan, Patna 800007, Bihar, India

Tel +9l 06I2 $263 \mid 565$

Fax +9| 06I2 2634379

Email drpradeep.das@gmail.com

\begin{abstract}
New treatments for visceral leishmaniasis, caused by Leishmania donovani, are needed to overcome sustained toxicity, cost, and drug resistance. The aim of this study was to evaluate the therapeutic effects of 2-nitro- $N$-(pyridin-2-ylmethyl)benzenesulfonamide (2NB) against promastigote and amastigote forms of $L$. donovani and examine its effect in combination with amphotericin $\mathrm{B}(\mathrm{AmB})$ against $\mathrm{AmB}$-resistant clinical isolates. Effects were assessed against extracellular promastigotes in vitro and intracellular amastigotes in L. donovani-infected macrophages. Levels of inducible nitric oxide and Th1 and Th2 cytokines were measured in infected 2NB-treated macrophages, and levels of reactive oxygen species and NO were measured in 2NB-treated macrophages. $2 \mathrm{NB}$ was active against promastigotes and intracellular amastigotes with $50 \%$ inhibitory concentration values of $38.5 \pm 1.5 \mu \mathrm{g} / \mathrm{mL}$ and $86.4 \pm 2.4 \mu \mathrm{g} / \mathrm{mL}$, respectively. $2 \mathrm{NB}$ was not toxic to macrophages. Parasite titer was reduced by $>85 \%$ in infected versus uninfected macrophages at a $2 \mathrm{NB}$ concentration of $120 \mu \mathrm{g} / \mathrm{mL}$. The parasiticidal activity was associated with increased levels of Th1 cytokines, NO, and reactive oxygen species. Finally, 2NB increased the efficacy of AmB against AmB-resistant L. donovani. These results demonstrate $2 \mathrm{NB}$ to be an antileishmanial agent, opening up a new avenue for the development of alternative chemotherapies against visceral leishmaniasis.
\end{abstract}

Keywords: visceral leishmaniasis, AmB resistance, benzenesulfonamide, ROS, NO, Th1/ Th2 cytokines

\section{Introduction}

Currently, $>350$ million people in 98 countries are at risk of leishmaniasis, with approximately half a million new cases of visceral leishmaniasis (VL) diagnosed annually and $>50,000$ associated deaths. More than $90 \%$ of VL cases occur in just six countries, namely, India, Nepal, Bangladesh, Sudan, Ethiopia, and Brazil., ${ }^{1,2}$ There are no effective vaccines available against VL; thus, treatment relies solely on chemotherapy. Current health practice depends on a limited number of drugs (such as miltefosine and aminoglycosides) that have issues of toxicity, long-dose regimens, high cost, and the need for parenteral administration. ${ }^{3}$ The toxicity of miltefosine includes its teratogenic potential and its long half-life ( $\sim 150$ hours), which may facilitate the emergence of resistance, ${ }^{4,5}$ and aminoglycosides-related adverse effects, including elevated hepatic transaminases, ototoxicity, nephrotoxicity, and pain at the injection site. ${ }^{6,7}$ Drug efficacy against different clinical isolates is variable, and the emergence of acquired resistance to the pentavalent antimonials is a major concern, particularly in the state of Bihar, India, where $64 \%$ of cases show resistance to antimonials. Similarly, 
emerging resistance to amphotericin $B(A m B)$ in Bihar emphasizes the need for new and effective treatments. ${ }^{3,8,9}$

Sulfonamide drugs have a broad-spectrum application through their antibacterial, ${ }^{10}$ anticarbonic anhydrase, ${ }^{11,12}$ and antiproton activities. ${ }^{13,14}$ In this study, 2-nitro- $N$-(pyridin2-ylmethyl)benzenesulfonamide (2NB) (CID 779413), a benzenesulfonamide derivative, which is a chemokine receptor-binding heterocyclic compound, was used. ${ }^{15}$ Benzenesulfonamides are effective in case of a proliferative disease, such as cancer, ${ }^{16}$ and are also effective against Leishmania tropica, Toxoplasma, Entamoeba histolytica, ${ }^{17-19}$ and Plasmodium falciparum. ${ }^{20}$ Similarly, anticancer drugs, such as miltefosine, and some protein kinase inhibitors ${ }^{21}$ are effective against VL. Therefore, 2NB was selected and tested against Leishmania donovani. Sulfonamide anilide is an inhibitor of histone deacetylase. ${ }^{22} \mathrm{We}$ have previously shown that high level of silent information regulator 2 (Sir2) of L. donovani, a histone deacetylase, is associated with AmB resistance in parasites. ${ }^{23}$ This led us to predict that our compound of interest $2 \mathrm{NB}$ may reverse AmB resistance in combination with AmB through inhibition of Sir2 activity.

More than 100 sulfonamide-containing drugs are currently on the market. ${ }^{24}$ Therefore, the use of $2 \mathrm{NB}$ could provide a rapid and cost-effective approach to antileishmanial drug discovery. Here, we tested the therapeutic potential of $2 \mathrm{NB}$ against Leishmania promastigotes and also the intracellular amastigotes via infected peritoneal mouse macrophages. We also evaluated the toxicity level of $2 \mathrm{NB}$ on peritoneal macrophages. Therefore, the major objective of this investigation is to evaluate the antileishmanial effect of $2 \mathrm{NB}$ and its potential to be used in combination with AmB against AmB-resistant clinical isolates.

\section{Materials and methods Experimental animals}

Female BALB/c mice 6-8 weeks old were obtained from breeding stocks maintained at the animal husbandry of Rajendra Memorial Research Institute of Medical Sciences (RMRIMS), Patna, India. Mice were injected with 4\% starch and sacrificed after 48 hours. Peritoneal macrophages were isolated and seeded onto well plates according to the experimental protocol described in (Cell cytotoxicity assay) section. Macrophages were infected with L. donovani promastigotes, and the effect of $2 \mathrm{NB}$ was tested on intracellular amastigotes. A total of 12 mice were used to obtain peritoneal macrophages for all the experiments.

\section{Ethical statement}

For animal use, the procedures used were reviewed and approved by the Animal Ethical Committee, RMRIMS,
Indian Council of Medical Research (ICMR). The RMRI (ICMR) follows "The Guide for the Care and Use of Laboratory Animals," 8th edition, by the Institute for Laboratory Animal Research. This study was approved by the Institutional Ethical Committee of RMRIMS.

\section{Compound}

2NB (CID 779413), a derivative of sulfonamide drug, was purchased from Asinex (Moscow, Russia). The compound 2NB (Figure 1) was dissolved in distilled water $\left(\mathrm{dH}_{2} \mathrm{O}\right)$ at a stock concentration of $5 \mathrm{mg} / \mathrm{mL}$.

\section{Parasite culture}

L. donovani promastigotes, AG83 (MHOM/IN/1983/AG83), were maintained in M199 medium (Thermo Fisher Scientific, Waltham, MA, USA) supplemented with $10 \%$ fetal bovine serum (FBS) at $25^{\circ} \mathrm{C}$ in a BOD incubator. Parasites were subcultured every 7 days, and only stationary-phase cultures were used for experiments.

AmB-resistant and-sensitive clinical isolates of L. donovani (used in our previous study) $)^{9,23}$ of VL were obtained from the splenic aspirates of $\mathrm{AmB}$ responder and nonresponder patients of the indoor ward facility of the RMRIMS, Patna, India, ${ }^{9}$ and were grown in Roswell Park Memorial Institute (RPMI)-1640 (Thermo Fisher Scientific) medium ( $\mathrm{pH} 7.4$ ), supplemented with $10 \%$ FBS (Thermo Fisher Scientific) and 1\% of penicillin $(50 \mathrm{U} / \mathrm{mL})$ and streptomycin $(50 \mathrm{mg} / \mathrm{mL})$ solution (SigmaAldrich Co., St Louis, MO, USA) at $250^{\circ} \mathrm{C}$ and maintained further under drug pressure. ${ }^{9}$

The resistant and sensitive nature of the parasites was confirmed by in vitro and ex vivo (macrophage infection) assay as described earlier by our group., ${ }^{9,23}$ Briefly, in in vitro drug sensitivity assay, $2 \times 10^{6}$ parasites were incubated with different concentrations of $\mathrm{AmB}$, and the viability of the cells was evaluated either by counting the viable cells

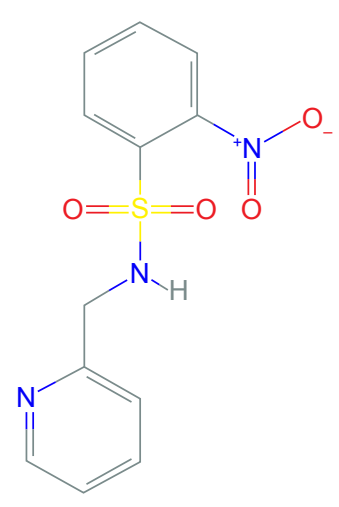

Figure I Molecular structure of 2-nitro- $N$-(pyridin-2-ylmethyl)benzenesulfonamide (2NB). 
in a hemocytometer (Rohem, Nashik, India) by the trypan blue (Sigma-Aldrich Co.) $(0.5 \mathrm{mg} / \mathrm{mL})$ exclusion method or by 3-(4,5-dimethylthiazol-2-yl)-2,5-diphenyl tetrazolium bromide (MTT) assay, and the $50 \%$ lethal doses $\left(\mathrm{LD}_{50}\right)$ were determined for both the AmB-resistant and AmB-sensitive strains. ${ }^{9,23}$ Briefly, in ex vivo drug sensitivity assay, ${ }^{9,23}$ adhered macrophages (THP1 cells) were infected with parasites at a ratio of 1:10 (macrophages:parasite), and AmB at different concentrations was then added to the infected macrophages and incubated for 48 hours. The number of viable amastigotes per 100 macrophages was counted under the microscope after Giemsa staining, and the $\mathrm{LD}_{50}$ values for both resistant and sensitive parasites were calculated. ${ }^{9,23}$

\section{Antileishmanial activity of 2 NB (in vitro) and determination of $\mathrm{IC}_{50}$}

In vitro drug sensitivity was performed by incubating $2 \times 10^{6}$ parasites in RPMI-1640 medium (supplemented with 10\% FBS) with indicated different concentrations of 2NB at 1-day intervals for 3 consecutive days. Parasites were not treated with $2 \mathrm{NB}$ in the control experimental set. The viability of the parasites was evaluated using MTT assay, ${ }^{9}$ where the conversion of MTT to formazan by mitochondrial enzymes served as an indicator of cell viability. The amount of formazan produced was directly proportional to the number of metabolically active cells. The $50 \%$ inhibitory concentration $\left(\mathrm{IC}_{50}\right)$ was determined after analyzing with MS Excel ${ }^{\mathrm{TM}}$ and Prism ${ }^{\mathrm{TM}}$.

\section{Inhibitor assay of AmB-resistant parasites by 2 NB}

As used in our previous work, for this experiment, three AmB-resistant and three AmB-sensitive parasites were used. $^{23} 2 \mathrm{NB}$ was added at a concentration of $20 \mu \mathrm{g} / \mathrm{mL}$ to AmB-resistant and -sensitive parasites and incubated for 4 hours at $23^{\circ} \mathrm{C}$ in a BOD incubator. The parasites were subsequently washed with phosphate-buffered saline $(\mathrm{pH}$ 7.2) and treated with $A m B$. $L_{50}$ values of $A m B$ were then determined. For positive control, known inhibitor of Sir2, that is, sirtinol, was also used to inhibit the parasites.

\section{Determination of enzyme activity (deacetylase activity) of Sir2 in 2NB- inhibited AmB-resistant and -sensitive parasites}

Total intracellular nicotinamide adenine dinucleotide $\left(\mathrm{NAD}^{+}\right)$-dependent deacetylase activity of Sir2 was measured for wild-type, 2NB-treated, and sirtinol (known inhibitor of Sir2)-treated parasites according to our previous work ${ }^{23}$ using
SIRT1/Sir2 deacetylase fluorometric assay kit (CycLex). Briefly, total cellular extracts were prepared and used as a cofactor for purified recombinant $L$. donovani SIR2 protein (LdSir2RP). In control reaction, $\mathrm{NAD}^{+}$of the kit was used as a cofactor for the purified LdSir2RP. The results were recorded in an LS55 Spectrofluorimeter (PerkinElmer Inc., Waltham, MA, USA). The results were expressed as the rate of reaction for the first 20 minutes when there was a linear correlation between the fluorescence and the period of time.

\section{Cell cytotoxicity assay}

This assay was performed as described previously, ${ }^{9}$ with some modifications. Briefly, primary macrophages that were harvested from starch-induced peritoneal exudates in BALB/c mice were seeded $\left(10^{4}\right.$ cells/well $)$ in a 96 -well plate with different concentrations of $2 \mathrm{NB}$. After 48 hours of incubation, the medium was removed, $200 \mu \mathrm{L}$ of fresh supplemented medium and $20 \mu \mathrm{L}$ of Alamar blue (Sigma-Aldrich Co.) were added, and the absorbance was measured at $550 \mathrm{~nm}$. There were three replicates in each test, and the data reported herein are the mean \pm standard deviation of the three experiments.

\section{Activity of 2 NB against $L$. donovani- infected macrophages}

Starch-induced peritoneal exudate-harvested macrophages from BALB/c mice were seeded in 16-well glass slides and infected with Leishmania promastigotes (at a ratio of 1:10= macrophages:parasite) as previously described. ${ }^{9,25}$ The infected macrophages were exposed to $2 \mathrm{NB}$ for 48 hours, after which the percentage of infected macrophages and the amastigotes per 100 macrophages was determined microscopically after Giemsa staining ${ }^{25,26}$ followed by $\mathrm{IC}_{50}$ calculation as described previously. ${ }^{9}$

\section{Semi-quantitative reverse transcription- polymerase chain reaction}

The messenger RNA level of inducible nitric oxide (iNOS) was performed by isolating the total RNA from 2NBtreated/untreated peritoneal macrophages using Trizol method. Reverse transcription was performed using an anchored oligo(dT) (H-dT11M, where M represents A, C, or G; GenHunter, Nashville, TN, USA). ${ }^{9}$ The synthesized complementary DNAs were amplified by polymerase chain reaction (PCR) for $i N O S$ gene. The PCR product was run on $1.5 \%$ agarose gel, stained with ethidium bromide, and finally documented and quantified using the Bio-Rad gel documentation system and the associated Quantity One software. PCR product was normalized with respect 
to the glyceraldehyde-3-phosphate dehydrogenase reverse transcription PCR product.

\section{Cytokine production}

The ability of $2 \mathrm{NB}$ to induce the production of the cytokines was tested using peritoneal macrophage cells. These cells were cultured in 24-well plates, in two conditions, 1) infected with L. donovani at a ratio of ten parasites:one macrophage and 2) no infection and incubated for $6-8$ hours at $37^{\circ} \mathrm{C}$ in $5 \% \mathrm{CO}_{2}$. Free parasites were removed by washing with phosphate-buffered saline ( $\mathrm{pH} 7.2$ ), and the cultures were maintained in RPMI1640 medium supplemented with $10 \%$ FBS for 24 hours at $37^{\circ} \mathrm{C}$ in $5 \% \mathrm{CO}_{2}$. After incubation, the infected macrophages were treated with $100 \mu \mathrm{g} / \mathrm{mL}$ of $2 \mathrm{NB}$. After 48 hours of treatment, the production of Th1 cytokines (interleukin [IL]-12 and tumor necrosis factor [TNF]- $\alpha$ ) and Th2 cytokines (IL-10 and transforming growth factor [TGF]- $\beta$ ) was measured in the cell culture supernatants using BioVision enzyme-linked immunosorbent assay (ELISA) kit according to the manufacturer's instructions. All the assays were performed in triplicates.

\section{Measurement of reactive oxygen species (ROS)}

To measure the level of ROS, the cell permeable probe $\mathrm{H}_{2}$ DCFDA (Sigma-Aldrich Co.) was used as described previously. ${ }^{27}$ Infected 2 NB-treated/untreated peritoneal macrophages were incubated with $\mathrm{H}_{2} \mathrm{DCFDA}(2 \mathrm{mg} / \mathrm{mL})$ at room temperature for 20 minutes in the dark. Relative fluorescence was measured in a PerkinElmer Inc., LS55 Spectrofluorometer at an excitation wavelength of $508 \mathrm{~nm}$ and emission wavelength of $529 \mathrm{~nm}$. Fluorometric measurements were made in triplicate and expressed as mean fluorescence intensity units.

\section{Quantification of NO}

NO was quantified by the accumulation of nitrite in macrophage culture supernatants, and nitrite was detected by the Griess reaction as described previously. ${ }^{28}$

\section{Statistical analysis}

All the experiments were conducted at least in triplicate, and the results are expressed as mean \pm standard deviation of the three experiments, and the data were statistically analyzed by a single analysis of variance test. A $P$-value of $<0.01$ was considered significant.

\section{Results and discussion}

The commercially available compound 2NB was tested against L. donovani promastigotes (MHOM/IN/1983/AG83) in vitro and intracellular amastigotes cultured in mouse peritoneal macrophages. The in vitro assay revealed that $2 \mathrm{NB}$ showed significant activity against $L$. donovani promastigotes (Figure 2), with an $\mathrm{IC}_{50}$ value of $38.5 \pm 1.5 \mu \mathrm{g} / \mathrm{mL}$. Treatment of promastigotes with $2 \mathrm{NB}$ demonstrated a dose-dependent inhibition of the parasite growth (Figure 2), indicating parasite-killing ability.

The bacteriostatic sulfonamide ${ }^{29}$ is active against Toxoplasma and Entamoeba. ${ }^{19,20}$ Typically, sulfonamides suppress bacterial growth by competitive blockade of paraaminobenzoic acid to prevent the synthesis of folic acid. Since, in humans, folate accumulation takes place through the diet, ${ }^{30}$ sulfonamide has no effect on human cellular machinery. Although antifolates such as pyrimethamine, sulfa drugs, and trimethoprim are effective against protozoan infectious diseases, ${ }^{31-33}$ antifolate chemotherapy has not been achieved against Leishmania infections. ${ }^{34}$ Here, we found potential antileishmanial activity by $2 \mathrm{NB}$, consistent with sulfonamide activity against L. tropica. ${ }^{18}$ This suggests a different antileishmanial target in Leishmania promastigotes than the folate biosynthetic pathway, possibly the carbonic anhydrase as reported in L. chagasi. ${ }^{35}$

$2 \mathrm{NB}$ in combination with $\mathrm{AmB}$ reverses the resistant property of the AmB-resistant parasites. ${ }^{23}$ In our previous study, we showed that histone deacetylase enzyme, Sir2, was highly overexpressed in AmB-resistant parasites compared to the sensitive parasites, and this upregulation

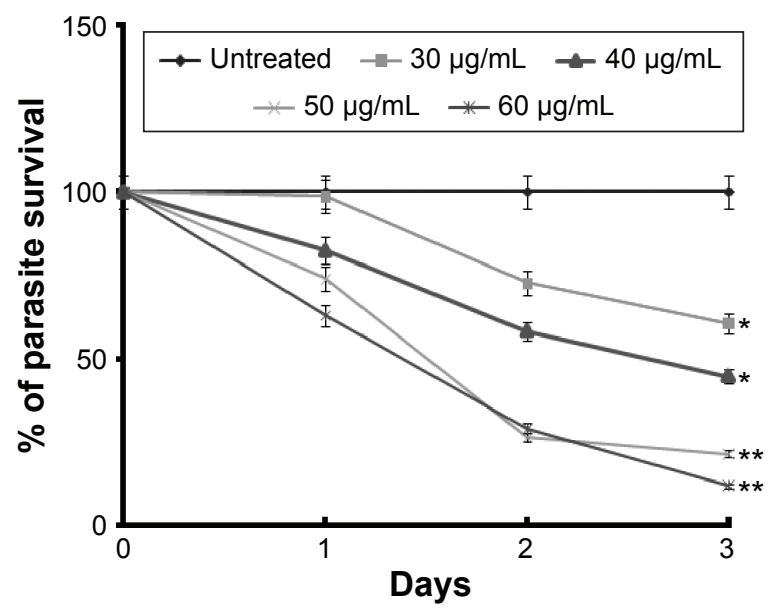

Figure 2 Activity of 2-nitro- $N$-(pyridin-2-ylmethyl)benzenesulfonamide (2NB) against Leishmania donovani promastigotes.

Notes: Promastigotes were treated with indicated concentrations of compound $2 \mathrm{NB}$ and the \% of cell viability was checked at 24-hour interval for 3 consecutive days. Cell viability was reduced in a dose-dependent manner. In each test, there were three replicates, and the data are mean $\pm S D$ at each time point. $* P<0.01$ for $30 \mu \mathrm{g} / \mathrm{mL}$ and $40 \mu \mathrm{g} / \mathrm{mL}$ and $* * P<0.00 I$ for $50 \mu \mathrm{g} / \mathrm{mL}$ and $60 \mu \mathrm{g} / \mathrm{mL}$ versus untreated control at day 3 .

Abbreviation: SD, standard deviation. 

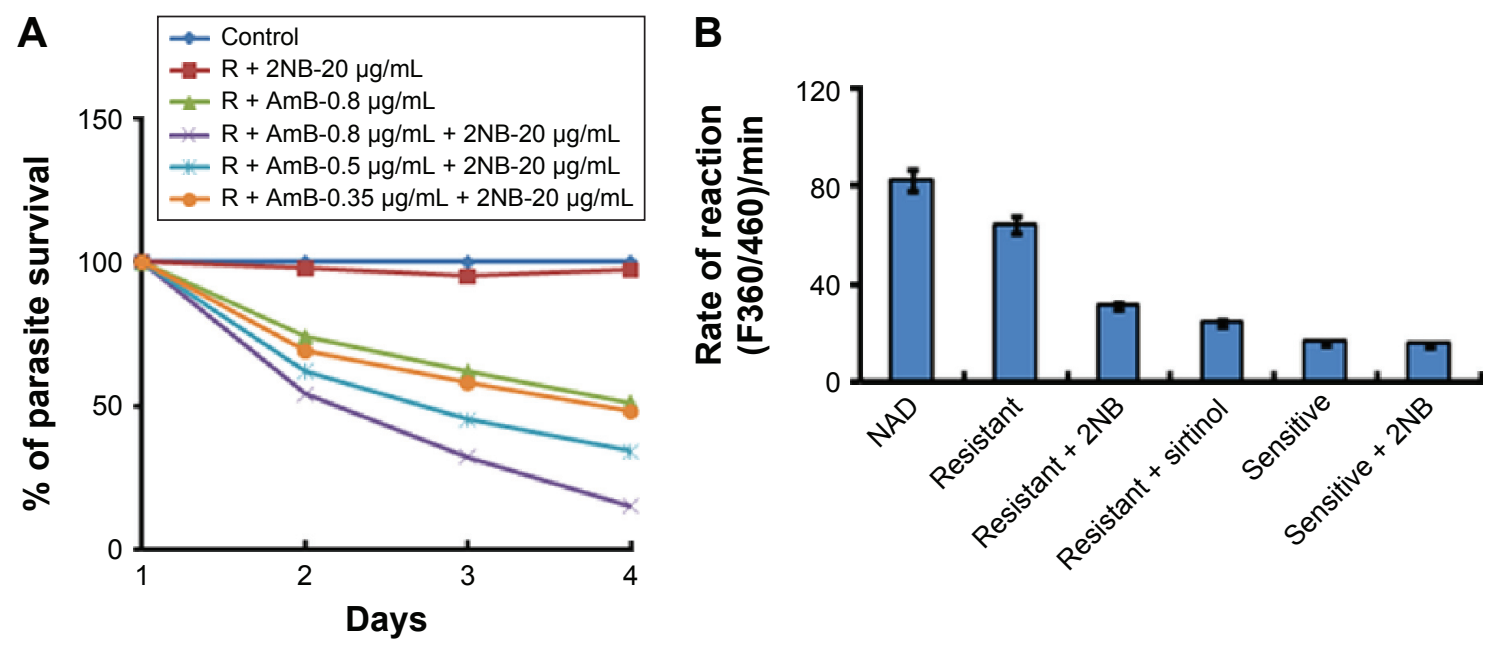

Figure 3 Modulation of AmB-resistant property of AmB-resistant L. donovani by our compound of interest $2 \mathrm{NB}$.

Notes: (A) Determination of $\mathrm{LD}_{50}$ of $\mathrm{AmB}$ of the resistant parasites after inhibition with the compound $2 \mathrm{NB}$. The concentration of $2 \mathrm{NB}(20 \mu \mathrm{g} / \mathrm{mL}) \mathrm{used}$ for inhibition study was not toxic to the parasites. The $\mathrm{LD}_{50}$ of $\mathrm{AmB}$ of the resistant parasites was decreased $\sim 2.5$-fold when pretreated with $2 \mathrm{NB}$. (B) Measurement of the deacetylase activity of Sir2 of resistant parasites after inhibition with $2 \mathrm{NB}(20 \mu \mathrm{g} / \mathrm{mL})$. NAD $(0.6 \mathrm{mM})$ was used as the cofactor of the enzyme and was used to show the activity of the Sir2 in the parasites. Sirtinol ( $15 \mu \mathrm{M})$, known inhibitor of Sir2, was used as a positive control.

Abbreviations: AmB, amphotericin B; 2NB, 2-nitro-N-(pyridin-2-ylmethyl)benzenesulfonamide; $\mathrm{LD}_{50}$, $50 \%$ lethal dose; Sir2, silent information regulator 2; min, minutes; $R, A m B$ resistant; NAD, nicotinamide adenine dinucleotide.

of Sir2 was associated with AmB-resistant property of the parasites. ${ }^{23}$ Here, treatment of AmB-resistant parasites with 2NB decreased the $\mathrm{LD}_{50}$ of $\mathrm{AmB} \sim 2.5$-fold compared to the untreated resistant parasites (Figure 3A; Table 1). There was no change in the $\mathrm{LD}_{50}$ of $\mathrm{AmB}$ for the sensitive parasites after inhibition with the compound 2NB (Table 1). The deacetylase activity of Sir2 of AmB-resistant parasites was found to be significantly higher compared to the sensitive parasites, which correlates our previous report of upregulation of Sir2 in AmB-resistant parasites. ${ }^{23}$ It was reported that sulfonamide anilides inhibit the histone deacetylase enzyme, ${ }^{22}$ and in this study, 2NB, being a sulfonamide compound, reduced the deacetylase activity of Sir2 of AmB-resistant

Table I Reversion of resistant and sensitive phenotype by inhibition with the compound, 2-nitro- $N$-(pyridin-2-ylmethyl) benzenesulfonamide (2NB), in in vitro AmB sensitivity assay

\begin{tabular}{lll}
\hline Experimental sets & LD $_{50}$ value $(\mu \mathrm{g} / \mathrm{mL})$ & Fold change $^{\mathrm{a}}$ \\
\hline Wild-type resistant $+\mathrm{AmB}$ & $0.80 \pm 0.014$ & - \\
Wild-type sensitive $+\mathrm{AmB}$ & $0.11 \pm 0.01 \mathrm{I}$ & - \\
$2 \mathrm{NB}+$ resistant $+\mathrm{AmB}$ & $0.35 * \pm 0.042$ & $\sim 2.5$ (decrease) \\
Sirtinol + resistant $+\mathrm{AmB}$ & $0.22 * \pm 0.038$ & $\sim 3.6$ (decrease) \\
$2 \mathrm{NB}+$ sensitive $+\mathrm{AmB}$ & $0.10 \pm 0.009$ & - \\
\hline
\end{tabular}

Notes: $\mathrm{LD}_{50}$, lethal dose 50 means the concentration of drug at which $50 \%$ cells will be dead or $50 \%$ cells will be viable. Sirtinol (at a concentration of I5 $\mu \mathrm{M}$ ), inhibitor of Sir2, was used as a positive control. After treatment with $2 \mathrm{NB}$, the resistant property of the AmB-resistant parasites was reversed as the $L D_{50}$ value of $A m B$ of the resistant parasites was decreased $\sim 2.5$-fold. Asterisk $(*)$ denotes that the data are significantly different from wild-type resistant and sensitive strains, $P<0.05$. ${ }^{\text {a }}$ Fold increase or decrease from the $L D_{50}$ value of the wild-type sensitive and resistant strains. $L_{50}$ values are represented as concentration of $A m B \pm$ standard deviation.

Abbreviations: AmB, amphotericin B; Sir2, silent information regulator 2.
L. donovani parasites $\sim 2.6$-fold compared to the untreated resistant parasites (Figure $3 \mathrm{~B}$ ). Therefore, these results demonstrate that our compound of interest $2 \mathrm{NB}$ can inhibit the deacetylase activity of Sir2 as evidenced by Figure 3 and can consequently reverse the AmB-resistant property of resistant parasites (Table 1), which correlates with our previous study. ${ }^{23} 2 \mathrm{NB}$ at a concentration of $20 \mu \mathrm{g} / \mathrm{mL}$ had no significant toxic effect on the untreated resistant and sensitive parasites (data not shown). So, our compound of interest $2 \mathrm{NB}$ in combination with AmB may increase the efficacy of the $\mathrm{AmB}$ and may be used in combination with $\mathrm{AmB}$ for the treatment of resistant cases.

In order to test the efficacy of the drug on intracellular amastigotes, peritoneal macrophages were infected with L. donovani and treated with different concentrations of $2 \mathrm{NB}$. The number of amastigotes was counted microscopically on 100 macrophages per sample, and the results were expressed as percent of reduction of the infection rate in comparison to that of the controls (Figure 4B). ${ }^{23,24} 2 \mathrm{NB}$ was found to inhibit amastigote growth in a dose-dependent manner (Figure 4) with an $\mathrm{IC}_{50}$ value of $86.4 \pm 2.4 \mu \mathrm{g} / \mathrm{mL}$ and reduced the parasite burden in infected macrophages by $>85 \%(P<0.001)$ as compared to untreated controls (Figure 4C). Furthermore, up to $2 \mathrm{NB}$ concentration of $150 \mu \mathrm{g} / \mathrm{mL}$, no cytotoxicity was observed against the murine macrophages, which indicates the selectivity of $2 \mathrm{NB}$ against amastigotes compared with mammalian cells as evaluated by qualitative microscopic examination (Figure 4A). To the best of our knowledge, this is the first report of 

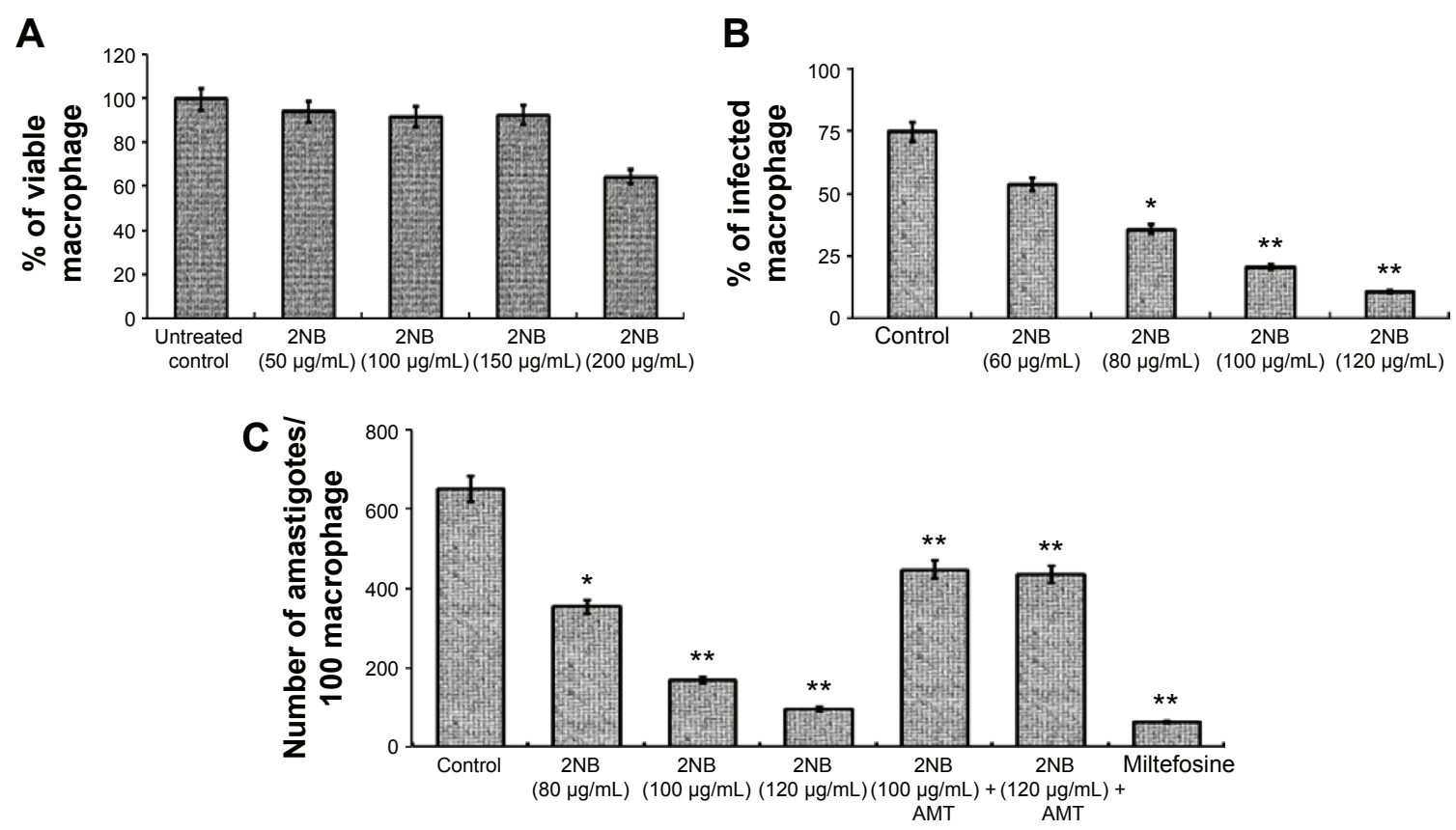

Figure 4 Macrophage infection assay (ex vivo) to determine the effect of compound 2NB on intracellular amastigotes.

Notes: (A) Evaluation of macrophage toxicity of 2NB. Adhered murine peritoneal macrophages were incubated with indicated concentrations of 2 NB. Cell viability was evaluated using alamar blue. (B) Determination of the numbers of macrophages infected with at least one amastigote, and then the percentage of infected macrophages was determined. (C) Peritoneal macrophages were infected with $L$. donovani promastigotes at a ratio of I:I0= acrophages:parasite. Infected macrophages were then treated with indicated concentrations of 2NB for 48 hours. In another set of experiments, AMT (I5 mM) (as an NO synthase inhibitor) was given along with 2 NB. Intracellular amastigotes number was determined by Giemsa staining. The number of intracellular amastigotes per 100 macrophages was determined microscopically. The bar diagrams show the number of parasites per 100 peritoneal macrophages. Miltefosine $(2 \mu \mathrm{M})$ was used as a positive control. In each test, there are three replicates, and the data are mean \pm SD at each time point. Asterisk $(* * *)$ denotes $(* P<0.0$ I, $* * P<0.00$ I $)$ that the data are significantly different from the control.

Abbreviations: 2NB, 2-nitro-N-(pyridin-2-ylmethyl)benzenesulfonamide; AMT, 2-amino-5,6-dihydro-6-methyl-4H-I,3-thiazine; SD, standard deviation.

antileishmanial activity of $2 \mathrm{NB}$ that can also reduce parasite burdens in infected macrophages.

ROS and NO, important macrophage-derived microbicidal molecules, are essential to control Leishmania infection. ${ }^{27,36}$ Therefore, the generation of ROS and NO in the culture supernatants of 2NB-treated and untreated L. donovani-infected macrophages was estimated. In infected 2NB-treated macrophages, the level of ROS was increased up to $\sim 5.4$-fold $(P<0.001)$ as compared to the infected control (Figure 5A). Similarly, 2NB increased NO generation in peritoneal macrophages in a dose- and time-dependent manner (Figure 5F) that was found to be maximal at 24 hours $\left(27.87+2.1 \mathrm{mM} / 10^{6}\right.$ cells) at a dose of $120 \mu \mathrm{g} / \mathrm{mL}$ of $2 \mathrm{NB}$ (Figure 5B). We then checked whether 2NB treatment could enhance the generation of $\mathrm{NO}$ in infected macrophages. Nitrite generation was markedly increased after 2NB $(120 \mu \mathrm{g} / \mathrm{mL})$ treatment in infected peritoneal macrophages up to $\sim 6$-fold $(P<0.001)$ as compared to untreated infected control (Figure 5B). Consequently, the mRNA level of $i N O S$ was increased $\sim 4$-fold $(P<0.001)$ with the treatment of $120 \mu \mathrm{g} / \mathrm{mL} 2 \mathrm{NB}$ in peritoneal macrophages infected with L. donovani (Figure 5C). Using an iNOS inhibitor, 2-amino-5,
6-dihydro-6-methyl-4H-1,3-thiazine (AMT), the rate of infection was measured to confirm the involvement of NO in the inhibition of intracellular amastigote multiplication by $2 \mathrm{NB}$. At 48 hours posttreatment, $15 \mathrm{mM}$ AMT markedly reduced ( $82 \%$ reduction in parasite clearance) the inhibitory effect of 2NB in ex vivo culture condition (Figure 4C). For the cure of VL, iNOS upregulation and subsequent release of nitrogen metabolites are essential. ${ }^{36,37}$ However, both reactive nitrogen and oxygen intermediates are important factors for the cure of VL. ${ }^{38,39}$ Significantly enhanced generation of ROS and NO in 2NB-treated macrophages further suggested the overall activated state of cells for successful elimination of parasite ex vivo.

We then investigated the role of immune system in parasite killing by $2 \mathrm{NB}$ in infected macrophage model. Macrophage-produced cytokines can affect the intracellular growth of Leishmania, and its infection results in impaired microbicidal machinery of macrophages as evidenced by modification of Th1/Th2 paradigm, resulting in parasite survival. ${ }^{40-43}$ Along with NO production, the level of IL-12 and TNF- $\alpha$ was also increased in 2NB-treated macrophages in a dose- and time-dependent manner, and maximum 
A

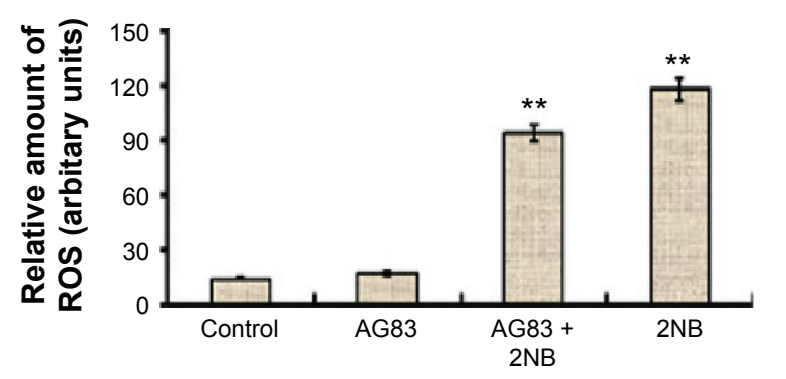

C

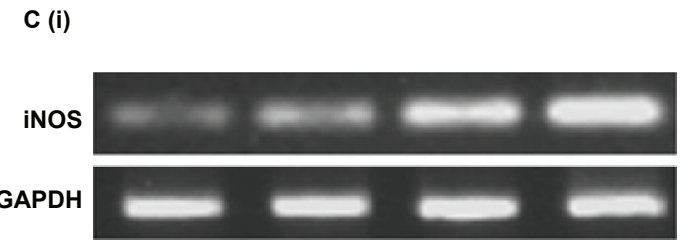

D

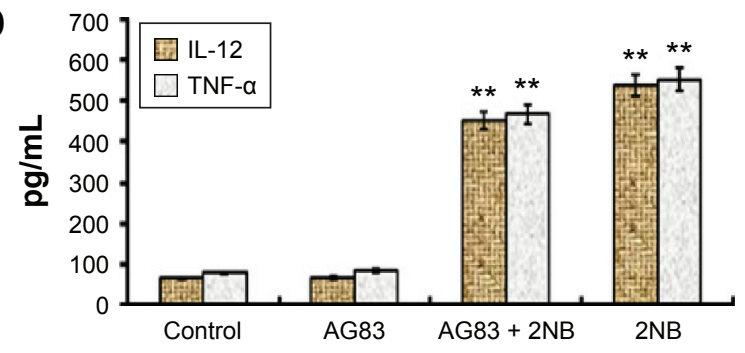

$\mathbf{F}$

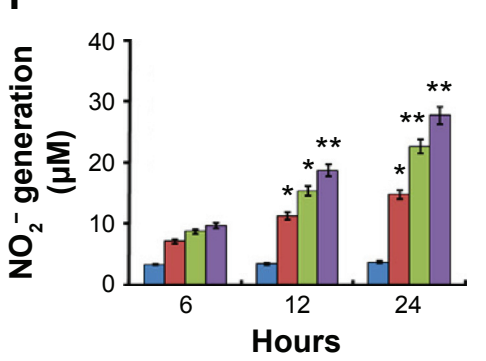

G
B
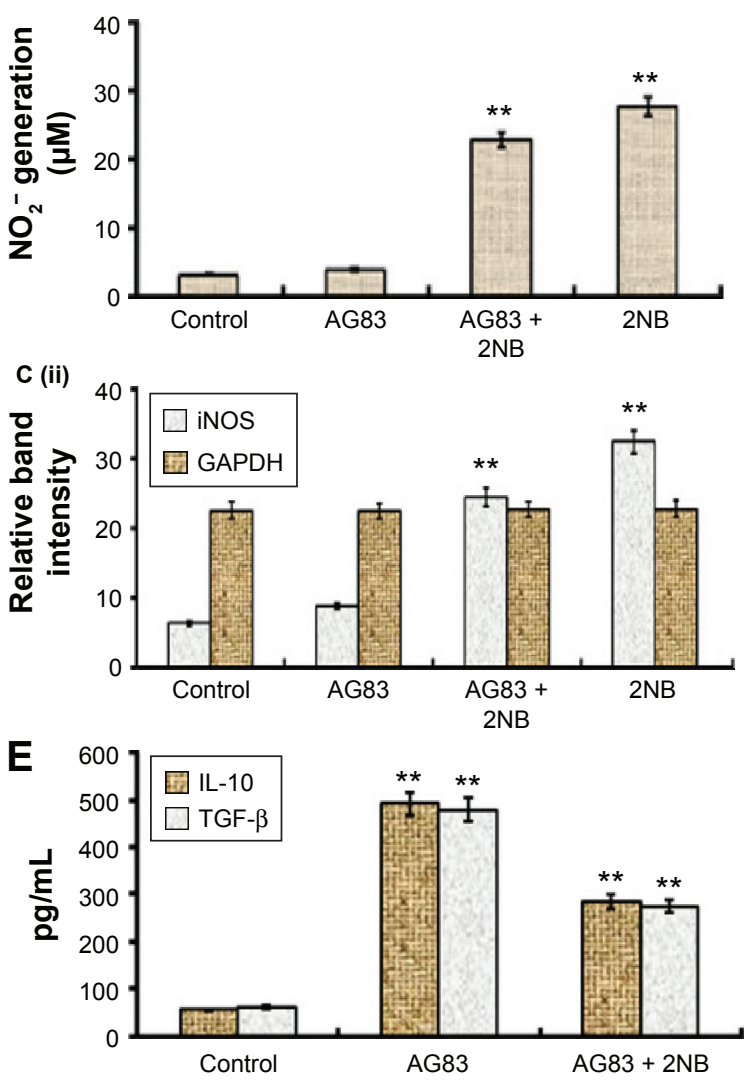

H

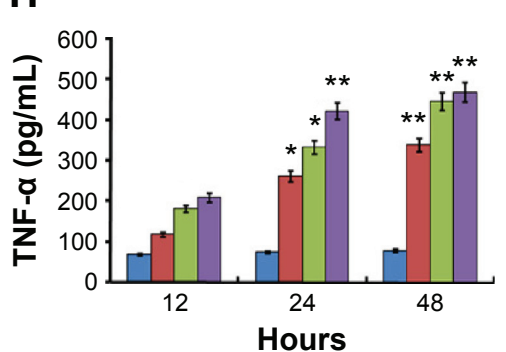

$\square$ Control $\square 80 \mu \mathrm{g} / \mathrm{mL} \quad \square 100 \mu \mathrm{g} / \mathrm{mL} \quad \square 120 \mu \mathrm{g} / \mathrm{mL}$

Figure 5 2NB treatment induces ROS and NO generation as well as modulates different cytokines response.

Notes: (A) 2NB induced generation of ROS from infected and uninfected peritoneal macrophages. ROS generation was measured by $\mathrm{H}_{2} \mathrm{DCFDA}$ probe. (B-E) Effect of $2 \mathrm{NB}$ on NO generation and cytokine response. Peritoneal macrophages from BALB/c mice were infected with $L$. donovani promastigotes (macrophage/parasite, I:I0). Noningested promastigotes were removed by washing, and macrophages were cultured for another 20 hours. Infected macrophages were then treated with $2 \mathrm{NB}$ (I $20 \mu \mathrm{g} / \mathrm{mL})$ for 24 hours. NO production (B) was determined by measuring the accumulation of nitrite in the culture medium by Griess reagent. iNOS expressions at mRNA (C) levels were evaluated by semiquantitative RT-PCR. GAPDH PCR product was used as loading control. C (i) shows the gel image and C (ii) shows the densitometric analysis of the band intensity. Peritoneal macrophages were treated with $2 \mathrm{NB}(120 \mu \mathrm{g} / \mathrm{mL})$ for 48 hours, and levels of IL- 12 and TNF- $\alpha$ (D) and IL- 10 and TGF- $\beta$ (E) in culture supernatants were determined by ELISA. Error bars represent mean \pm SD. The data shown are representative of three independent experiments. $* P<0.0 \mathrm{I}$ and $* * P<0.00 \mathrm{I}$ versus corresponding infected or uninfected control. (F-H) Time- and dose-dependent increase in the level of nitric oxide, IL-I2, and TNF- $\alpha$ after treatment with indicated concentrations of 2 NB. Bars marked AG83 refer to the infected macrophages.

Abbreviations: 2NB, 2-nitro-N-(Pyridin-2-ylmethyl)benzenesulfonamide; ROS, reactive oxygen species; iNOS, inducible nitric oxide; mRNA, messenger RNA; RT-PCR, reverse transcription-polymerase chain reaction; GAPDH, glyceraldehyde-3-phosphate dehydrogenase; IL, interleukin; TNF- $\alpha$, tumor necrosis factor $\alpha$; TGF- $\beta$, transforming growth factor- $\beta$; ELISA, enzyme-linked immunosorbent assay; SD, standard deviation.

induction of IL-12 and TNF- $\alpha$ was observed at 48 hours posttreatment (Figure $5 \mathrm{G}$ and $\mathrm{H}$ ) with a dose of $120 \mu \mathrm{g} / \mathrm{mL}$ of $2 \mathrm{NB}$. Therefore, in order to evaluate whether $2 \mathrm{NB}$ could modulate the infected macrophages for production of these pro- and anti-inflammatory cytokines, Th1 cytokines (IL-12, TNF- $\alpha$ ) and Th 2 cytokines (IL-10, TGF- $\beta$ ) levels were measured in supernatants from $L$. donovani-infected peritoneal macrophages, untreated or treated with $120 \mu \mathrm{g} / \mathrm{mL} 2 \mathrm{NB}$. 
In untreated $L$. donovani-infected macrophages, the level of IL-12 (68 $\pm 6.8 \mathrm{pg} / \mathrm{mL})$ and TNF- $\alpha(84 \pm 8.1 \mathrm{pg} / \mathrm{mL}) \mathrm{did}$ not appreciably change (Figure 5D). However, the levels of IL-10 (8.4-fold increase, $P<0.001)$ and TGF- $\beta$ (7.7-fold increase, $P<0.001)$ were increased robustly after infection (Figure 5E). In contrast, 2NB (120 $\mu \mathrm{g} / \mathrm{mL})$ treatment significantly increased the production of pro-inflammatory cytokines, IL-12 (6.64-fold increase, $P<0.001$ ), and TNF- $\alpha$ (5.57-fold increase, $P<0.001$ ) in infected macrophages (Figure 5D). In contrast, 2NB $(120 \mu \mathrm{g} / \mathrm{mL})$ treatment decreased the level of anti-inflammatory cytokines, IL-10 (58\% decrease, $P<0.01)$, and TGF- $\beta$ (56\% decrease, $P<0.01)$ in infected macrophages compared to the untreated infected macrophages (Figure 5E). In infected macrophages, $2 \mathrm{NB}$ treatment at a dose of $120 \mu \mathrm{g} / \mathrm{mL}$ resulted in reduced amastigotes survival by the induced production of disease-resolving Th1 (IL-12, TNF- $\alpha$ ) cytokines and decreased release of disease-promoting Th2 (IL-10, TGF- $\beta$ ) cytokines. It was observed that the production of IL-12 and TNF- $\alpha$ was increased in Leishmania-infected macrophages after treatment as compared with the untreated controls and untreated uninfected controls. Although the level of reduction of IL-10 and TGF- $\beta$ was not very high in infected macrophages following treatment, this might be explained by the sharp induction of IL-12 and TNF- $\alpha$ (anti-Leishmania cytokine).

\section{Conclusion}

In conclusion, we have shown for the first time that a benzenesulfonamide, 2NB, possesses leishmanicidal activity against $L$. donovani in both promastigote and intracellular amastigote forms at concentrations that are not toxic to the host. The lethal effects are associated with induction of disease-resolving Th1 cytokine response along with the generation of ROS and NO. 2NB also increases the efficacy of the AmB and reverses the AmB-resistant property of the resistant $L$. donovani in combination with AmB. Therefore, 2NB compounds or its analogs may in future be used alone or in combination with conventional drugs as an alternate chemotherapy for VL.

\section{Acknowledgments}

The work is supported by ICMR, India. We would like to thank Dr Mark JI Paine, Liverpool School of Tropical Medicine, UK, for critically reviewing this manuscript. MRD and BP are the joint first authors.

\section{Disclosure}

The authors report no conflicts of interest in this work.

\section{References}

1. Chappuis F, Sundar S, Hailu A, et al. Visceral leishmaniasis: what are the needs for diagnosis, treatment and control? Nat Rev Microbiol. 2007;5:873-882.

2. Dawit G, Girma Z, Simenew K. A review on biology. Epidemiology and public health significance of leishmaniasis. J Bacteriol Parasitol. 2013;4:2.

3. Tiuman TS, Santos AO, Ueda-Nakamura T, et al. Recent advances in leishmaniasis treatment. Int J Infect Dis. 2011;15:e525-e532.

4. Dube A, Singh N, Sundar S, Singh N. Refractoriness to the treatment of sodium stibogluconate in Indian kalaazar field isolates persists in in-vitro and in-vivo experimental models. Parasitol Res. 2005;96:216-223.

5. Ritmeijer K, Dejenie A, Assefa Y. A comparison of miltefosine and sodium stibogluconate for treatment of visceral leishmaniasis in an Ethiopian population with high prevalence of HIV infection. Clin Infect Dis. 2006;43:357-364.

6. Sundar S, Jha TK, Thakur CP, Sinha PK, Bhattacharya SK. Injectable paromomycin for visceral leishmaniasis in India. $N$ Engl $J$ Med. 2007;356:2571-2581.

7. Athanasiou LV, Batzias GC, Saridomichelakis MN, et al. Pharmacokinetics and tolerability of aminosidine after repeated administrations using an optimal dose regimen in healthy dogs and in dogs with leishmaniasis. Vet Parasitol. 2014;205:365-370.

8. Walker RG, Thomson G, Malone K, et al. High throughput screens yield small molecule inhibitors of Leishmania CRK3: CYC6 cyclindependent kinase. PLoS Negl Trop Dis. 2011;5:e1033.

9. Purkait B, Kumar A, Nandi N, et al. Mechanism of amphotericin B resistance in clinical isolates of Leishmania donovani. Antimicrob Agents Chemother. 2012;56:1031-1041.

10. Drews J. Drug discovery: a historical perspective. Science. 2000;287: 1960-1964.

11. Supuran CT, Scozzafava A. Carbonic anhydrase inhibitors and their therapeutic potential. Expert Opin Ther Pat. 2000;10:575-600.

12. Supuran CT, Scozzafava A, Casini A. Carbonic anhydrase inhibitors. Med Res Rev. 2003;23:146-189.

13. Ogden RC, Flexner CW. Protease Inhibitors in AIDS Therapy. New York: Marcel Dekker; 2001.

14. Supuran CT, Scozzafava A, Mastrolorenzo A. Bacterial proteases: current therapeutic use and future prospects for the development of new antibiotics. Expert Opin Ther Pat. 2001;11:221-259.

15. Bridger, Gary James, et al. assignee. Chemokine receptor binding heterocyclic compounds. United States US Patent No. 7022717 B2. $2006 \mathrm{Apr}, 4$.

16. Bashir R, Ovais S, Yaseen S, et al. Synthesis of some new 1, 3, 5 -trisubstituted pyrazolines bearing benzene sulfonamide as anticancer and anti-inflammatory agents. Bioorg Med Chem Lett. 2011;21: 4301-4305.

17. Senekji $\mathrm{H}$. The effect of sulfanilamide and trypaflavin on cultures of Leishmania tropica. J Infect Dis. 1940;66:111-112.

18. Sabin AB, Warren J. Therapeutic effectiveness of certain sulfonamides on infection by an intracellular protozoon (Toxoplasma). Exp Biol Med. 1942;51:19-23.

19. Rodaniche EC, Kirsner JB. The effect of sulfonamide compounds on the growth of Entamoeba histolytica in culture. J Parasitol. 1942; 28:441-449.

20. Andrews KT, Fisher GM, Sumanadasa SD, et al. Antimalarial activity of compounds comprising a primary benzene sulfonamide fragment. Bioorg Med Chem Lett. 2013;23:6114-6117.

21. Sanderson L, Yardley V, Croft SL. Activity of anti-cancer protein kinase inhibitors against Leishmania spp. J Antimicrob Chemother. 2014; 69:1888-1891.

22. Fournel M, Trachy-Bourget MC, Yan PT, et al. Sulfonamide anilides, a novel class of histone deacetylase inhibitors, are antiproliferative against human tumors. Cancer Res. 2002;62:4325-4330.

23. Purkait B, Singh R, Wasnik K, et al. Up-regulation of silent information regulator 2 (Sir2) is associated with amphotericin B resistance in clinical isolates of Leishmania donovani. J Antimicrob Chemother. 2015; 70:1343-1356. 
24. Smith DA, Jones RM. The sulfonamide group as a structural alert: a distorted story? Curr Opin Drug Discov Devel. 2008;11:72-79.

25. Yardley V, Croft SL. A comparison of the activities of three amphotericin B lipid formulations against experimental visceral and cutaneous leishmaniasis. Int J Antimicrob Agents. 2000;13:243-248.

26. Das S, Rani M, Pandey K, et al. Combination of paromomycin and miltefosine promotes TLR4-dependent induction of antileishmanial immune response in vitro. J Antimicrob Chemother. 2012;67:2373-2378.

27. Sharma A, Madhubala R. Ubiquitin conjugation of open reading frame F DNA vaccine leads to enhanced cell-mediated immune response and induces protection against both antimony-susceptible and-resistant strains of Leishmania donovani. J Immunol. 2009;183:7719-7731.

28. Kar S, Ukil A, Das PK. Signaling events leading to the curative effect of cystatin on experimental visceral leishmaniasis: involvement of ERK1/2, NF-KB and JAK/STAT pathways. Eur J Immunol. 2009;39: 741-751.

29. Henry RJ. The mode of action of sulfonamides. Bacteriol Rev. 1943; 7:175.

30. Madigan MT. Brock Biology of Microorganisms. 11th ed. SciELO Barcelona; 2005.

31. Peters W, Richards WH. Antimalarial Drugs. 1: Biological Background, Experimental Methods, and Drug Resistance. 2: Current Antimalarials and New Drug Developments. (Handbook of Experimental Pharmacology Volume 68 Nos. 1 \& 2). Springer-Verlag: Berlin Heidelberg; 1984.

32. Grossman PL, Remington JS. The effect of trimethoprim and sulfamethoxazole on Toxoplasma gondii in vitro and in vivo. Am J Trop Med Hyg. 1979;28:445-455.

33. McDougald LR. Chemotherapy of coccidiosis. In: Long PL, editor. The Biology of the Coccidia. Baltimore, MD: University Park Press; 1982. pp. 373-427.

34. Hardy L, Matthews W, Nare B, Beverley SM. Biochemical and genetic tests for inhibitors of Leishmania pteridine pathways. Exp Parasitol. 1997; 87:158-170.

35. Syrjanen L, Vermelho AB, de Almeida Rodrigues I, et al. Cloning, characterization, and inhibition studies of a $\beta$-carbonic anhydrase from Leishmania donovani chagasi, the protozoan parasite responsible for leishmaniasis. J Med Chem. 2013;56:7372-7381.
36. Basu R, Bhaumik S, Basu JM, Naskar K, De T, Roy S. Kinetoplastid membrane protein-11 DNA vaccination induces complete protection against both pentavalent antimonial-sensitive and-resistant strains of Leishmania donovani that correlates with inducible nitric oxide synthase activity and IL-4 generation: evidence for mixed Th1-and Th2-like responses in visceral leishmaniasis. J Immunol. 2005; 174:7160-7171.

37. Das L, Datta N, Bandyopadhyay S, Das PK. Successful therapy of lethal murine visceral leishmaniasis with cystatin involves up-regulation of nitric oxide and a favorable T cell response. J Immunol. 2001;166: 4020-4028.

38. Roach T, Kiderlen AF, Blackwell JM. Role of inorganic nitrogen oxides and tumor necrosis factor alpha in killing Leishmania donovani amastigotes in gamma interferon-lipopolysaccharide-activated macrophages from Lshs and Lshr congenic mouse strains. Infect Immun. 1991;59:3935-3944.

39. Gantt KR, Goldman TL, McCormick ML, et al. Oxidative responses of human and murine macrophages during phagocytosis of Leishmania chagasi. J Immunol. 2001;167:893-901.

40. Awasthi A, Mathur RK, Saha B. Immune response to Leishmania infection. Indian J Med Res. 2004;119:238-258.

41. Rogers KA, DeKrey GK, Mbow ML, Gillespie RD, Brodskyn CI, Titus RG. Type 1 and type 2 responses to Leishmania major. FEMS Microbiol Lett. 2002;209:1-7.

42. Souza AS, Giudice A, Pereira JM, et al. Resistance of Leishmania (Viannia) braziliensis to nitric oxide: correlation with antimony therapy and TNF- $\alpha$ production. BMC Infect Dis. 2010;10:209.

43. Anderson CF, Lira R, Kamhawi S, Belkaid Y, Wynn TA, Sacks D. IL-10 and TGF- $\beta$ control the establishment of persistent and transmissible infections produced by Leishmania tropica in C57BL/6 mice. J Immunol. 2008;180:4090-4097.
Drug Design, Development and Therapy

\section{Publish your work in this journal}

Drug Design, Development and Therapy is an international, peerreviewed open-access journal that spans the spectrum of drug design and development through to clinical applications. Clinical outcomes, patient safety, and programs for the development and effective, safe, and sustained use of medicines are a feature of the journal, which

\section{Dovepress}

has also been accepted for indexing on PubMed Central. The manuscript management system is completely online and includes a very quick and fair peer-review system, which is all easy to use. Visit http://www.dovepress.com/testimonials.php to read real quotes from published authors. 